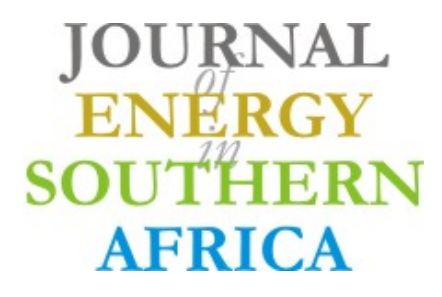

Volume 28 Number 2

\title{
Solar resource classification in South Africa using a new index
}

\section{Evans Zhandire}

University of KwaZulu-Natal, School of Engineering, Engineering Access, Howard College Campus, Durban 4041, South Africa.

\begin{abstract}
This paper introduces a solar resource index that responds to site-specific sky conditions resulting from stochastic movement and evolution of clouds. The developed solar resource classification index called probability of persistence $\left(P O P_{D}\right)$ had limited capabilities to distinguish persistent clear-sky conditions from persistent overcast-sky conditions. The metric proposed in this investigation, referred to as the solar utility index (SUI), seeks to extend the $P O P_{D}$ index to a simple enough index that can singly discriminate different states of a solar resource. It gives a measure of the fractional time during which a solar resource exhibits predefined characteristics over a specific time period not exceeding the time interval between sunrise and sunset. These solar resource qualities, which are user-defined, measure: (1) the fluctuation characteristic of the solar resource magnitude, and (2) the solar resource diffuse and beam composition. Values of the indexes computed over daily time intervals of 7:00-17:00 apparent solar time were tested for their solar resource classification qualities.
\end{abstract}

Five distinct classes using $K$-means clustering algorithm were identified for the solar radiation resource measured at eight stations in South Africa. The SUI was found to have superior solar resource discriminating and grouping abilities when compared with other indexes like $P O P_{D}$ and fractal dimension.

Keywords: fluctuation magnitude, K-means clustering, relative composition

\section{Highlights}

- Solar utility index, a new solar resource classification index was defined.

- Five classes of the solar resource in South Africa were identified.

- The five clusters showed reasonably homogeneous solar resource properties.

\footnotetext{
* Corresponding author: Tel: +27 31260 4101:
}

Email: Evans.zhandire@gmail.com 


\section{Introduction}

Solar energy is becoming an increasingly important component of the energy mix required to confront current global energy and environmental challenges. Detailed knowledge about its availability and variability over different time-scales are important for its exploitation to be cost-effective and efficient. Solar resource variability is primarily caused by earth-sun relative motion and movement and evolution of clouds. Variations induced by the apparent motion of the sun relative to the earth are visible on diurnal and seasonal scales, and can be predicted precisely from well-established astronomical equations [1, 2]. Variability caused by clouds is less predictable, and manifests as short-term temporal fluctuations that modulate the otherwise uniform astronomicallydriven diurnal irradiance profiles. These stochastic fluctuations vary in amplitude, persistence (duration), and frequency of occurrence [3]. Assessment of the solar resource therefore requires a statistical approach using appropriate statistical metrics that model the variation in solar resource magnitude under the influence of local stochastic weather influences over different time-scales. Several metrics that show varied solar resource discrimination capabilities exist in available literature. These include fractal dimension (FD) of daily profile of global horizontal irradiance (GHI) [4], daily clearness index probability distribution functions [5], granulometric size distribution of GHI [6], variability index (VI) [7] and daily probability of persistence POP $\mathrm{P}_{\mathrm{D}}$ [8]. The FD of $\mathrm{GHI}$ as proposed by Maafi and Harrouni [4] measures the amount of daily solar irradiance fluctuations that are due to changes in the state of the sky. Values of FD close to 1 indicate persistent skyconditions that are characteristic of either a clear day or an overcast day. These two extremes of the solar resource were distinguished by combining the FD with the daily clearness index $K_{T}$, to present a solar resource classifier that identified three classes of solar resource days using GHI data from two sites in Algeria [4]. The approach proposed by Soubdhan et al. [5] was that the classifier discriminates daily solar resource according to daily distribution histograms of instantaneous clearness indexes $k_{T}$. Four solar resource classes were identified at Guadeloupe, an island in the West Indies, from a year-long sample of irradiance data measured at a frequency of $1 \mathrm{~Hz}$. The membership of each class is subject to similarities in marginal probability density functions (pdfs) that are modelled using Dirichlet distribution functions from the daily histograms of clearness indexes $k_{T}(t)$. An elaborate five-step computational algorithm was used to implement the classification process [5]. Gastón-Romeo et al. [6], in another solar resource classification approach, proposed the use of granulometric size distribution curve, a mathematical morphology parameter, as a descriptor of the shape and dynamic of GHI daily curves. A sample of 609 solar radiation curves were partitioned into 4 classes using the partition around medoids clustering algorithm. Kang and Tam [8], in a more recent study, proposed a new metric: the daily probability of persistence $\mathrm{POP}_{\mathrm{D}}$. This metric measures the persistence of the normalised instantaneous magnitude of the GHI, i.e., instantaneous clearness index $k_{T}(t)$. Cases of consistently high or low magnitudes of $k_{T}(t)$ characteristic of clear-day or overcastday GHI time series will inevitably show similarly high $\mathrm{POP}_{\mathrm{D}}$ values [8]. These two extremes were differentiated by pairing the $\mathrm{POP}_{\mathrm{D}}$ with daily clearness index to form the K-POP method [8]. This method was used to classify the solar resource into 10 classes.

The present investigation focused on developing a solar resource metric that captures the cloudinduced fluctuations of the solar resource and that is able to classify the solar resource according to distinctive effects of weather induced effects. It extended $\mathrm{POP}_{\mathrm{D}}$ index to a simple enough index that can singly discriminate different states of a solar resource. This index is referred to as the solar utility index (SUI), which may also be considered as an indicator of the practical usefulness of a solar resource. The theoretical basis of the SUI and how it relates to the POP metric are discussed, followed by an outline of methodology to predict the properties of SUI and their subsequent demonstration, including solar resource classification capabilities.

\section{The solar utility index}

The $\mathrm{SUI}_{\tau}$ measures the fractional time when the solar resource has short-term fluctuation magnitudes and energy quality that satisfy a given set of conditions, for a solar resource available during a time $\tau$, between sunrise and sunset. The short-term fluctuation characteristic is given by $\left|\Delta k_{T}^{*}\right|$, a time series of absolute changes in $k_{T}^{*}$, the instantaneous clear sky index (CSI)) [9]. The CSI is simply a ratio of measured GHI to clear-sky global horizontal irradiance $\mathrm{GHI}_{\text {clear }}$, predicted by a suitable clear-sky model. A clear-sky model developed by Ineichen and Perez [10] and its MATLAB implementation developed by Sandia National Laboratory [11] were used to generate site-specific daily time series of $\mathrm{GHI}_{\text {clear }}$ in this investigation. The fluctuation characteristic $\left|\Delta k_{T}^{*}\right|$ for a time interval $\Delta t_{i}=t_{i+1}-t_{i}$ within a time span $\tau$, is given by Equation 1 .

$$
\left|\Delta \mathrm{k}_{\mathrm{Ti}}^{*}\right|=\left|\mathrm{k}_{\mathrm{T}}^{*}\left(\tau, \mathrm{t}_{\mathrm{i}}+\Delta \mathrm{t}\right)-\mathrm{k}_{\mathrm{T}}^{*}\left(\tau, \mathrm{t}_{\mathrm{i}}\right)\right|
$$

The energy quality is quantified by a new index called the relative composition index $(\mathrm{RCI})$, which, for a time interval $\Delta t_{i}=t_{i+1}-t_{i}$, is defined according to Equation 2. 


$$
\mathrm{RCI}_{\mathrm{i}}=\left[\mathrm{k}_{\mathrm{bd}}\left(\tau, \mathrm{t}_{\mathrm{i}}+\Delta \mathrm{t}\right)+\mathrm{k}_{\mathrm{bd}}\left(\tau, \mathrm{t}_{\mathrm{i}}\right)\right] / 2
$$

The $k_{b d}=(\mathrm{DHI}-\mathrm{BHI}) / \mathrm{GHI}$ is the instantaneous relative difference between the diffuse horizontal irradiance $\mathrm{DHI}$ and beam horizontal irradiance $\mathrm{BHI}$ at time $t_{i}$, within the time span $\tau$. These instantaneous indexes vary from a maximum value of 1 for overcast sky-conditions, through 0 when the beam and diffuse components are equal, to a negative value that is indicative of clear-sky conditions given by $\left(\mathrm{DHI}_{\text {clear }}-\mathrm{BHI}_{\text {clear }}\right) / \mathrm{GHI}_{\text {clear }}$. The solar utility index for a solar resource spanning the time period $\tau$ is then defined by Equation 3, which can be interpreted as the joint probability density function of a solar resource having fluctuation magnitude $\left|\Delta k_{T i}^{*}\right|$ less than or equal to $\Delta k_{t h}^{*}$ and relative composition index, $\mathrm{RCI}_{i}$ less than or equal to $\mathrm{RCI}_{t h}$ within a time span $\tau$.

$$
\begin{aligned}
& \operatorname{SUI}_{\tau}\left(\Delta k_{t h}^{*}, \mathrm{RCI}_{t h}\right)= \\
& \sum_{i=1}^{N-1} \Delta t_{i}\left(\mathrm{RCI}_{i} \leq \mathrm{RCI}_{t h} \cap\left|\Delta k_{T}^{*}\right| \leq \Delta k_{t h}^{*}\right) / \tau .
\end{aligned}
$$

where $\mathrm{RCI}_{t h}$ and $\Delta k_{t h}^{*}$ are reference values that define the threshold values of the $\mathrm{RCI}$ and fluctuation magnitude, respectively; and $N=\tau / \Delta t$ is the number of solar resource sampling points within the time span $\tau$. The $\mathrm{SUI}_{\tau}$ is notably a function of two marginal probabilities related to the cumulative distribution functions (CDFs) of the $\left|\Delta k_{T}^{*}\right|$ and $\mathrm{RCI}$ according to Equations 4 and 5.

$$
\begin{aligned}
& \operatorname{POP}_{\tau}^{*}\left(\Delta k_{t h}^{*}\right)=\mathrm{F}_{\left|\Delta k_{T}^{*}\right|}\left(\Delta k_{t h}^{*}\right)= \\
& \sum_{i=1}^{N} \Delta t_{i}\left(\left|\Delta k_{T}^{*}\right| \leq \Delta k_{t h}^{*}\right) / \tau \\
& \mathrm{PRC}_{\tau}\left(\mathrm{RCI}_{t h}\right)=\mathrm{F}_{\mathrm{RCI}}\left(\mathrm{RCI}_{t h}\right)= \\
& \sum_{i=1}^{N} \Delta t_{i}\left(\mathrm{RCI}_{i} \leq \mathrm{RCI}_{t h}\right) / \tau .
\end{aligned}
$$

The marginal probability distribution $\operatorname{POP}_{\tau}^{*}\left(\Delta k_{t h}^{*}\right)$ in Equation 4 is equivalent to the probability of persistence metric originally proposed by Kang and Tam [8]. The variation of $\operatorname{POP}_{\tau}^{*}\left(\Delta k_{t h}^{*}\right)$ with the threshold values $\Delta k_{\text {th }}^{*}$ can be obtained from $\mathrm{F}_{\left|\Delta k_{T}^{*}\right|}\left(\Delta k_{t h}^{*}\right)$, which is the CDF of $\left|\Delta k_{T i}^{*}\right|$ evaluated at $\Delta k_{\text {th }}^{*}$. Equation (5) defines a marginal probability distribution $\mathrm{PRC}_{\tau}\left(\mathrm{RCI}_{t h}\right)$ called probability of relative composition (PRC), which is the probability that $\mathrm{RCI}_{i} \leq \mathrm{RCI}_{t h}$. It follows that $\mathrm{F}_{\mathrm{RCI}_{i}}\left(\mathrm{RCI}_{t h}\right)$, which is the $\mathrm{CDF}$ of the $\mathrm{RCI}$, describes the variation of the $\mathrm{PRC}$ with the relative composition threshold. The PRC is related to the concept of utilisability, which is defined as the fraction of insolation incident on a collector's surface that is above a given threshold or critical value [12]. The functional relationship between the solar utility index and the two marginal probabilities $\operatorname{SUI}_{\tau}\left(\tau, \Delta t, \Delta k_{t h}^{*}, k_{b d t h}^{*}\right)=f\left(\operatorname{POP}_{\tau}^{*}\left(\Delta k_{t h}^{*}\right)\right.$,
$\left.\mathrm{PRC}_{\tau}\left(\mathrm{RCI}_{t h}\right)\right)$, depends on the probabilistic dependence of the two marginal events $\left|\Delta k_{T}^{*}\right| \leq \Delta k_{t h}^{*}$ and $\mathrm{RCI}_{i} \leq \mathrm{RCI}_{t h}$. If they were statistically independent, then their joint probability distribution $\mathrm{SUI}_{\tau}\left(\Delta k_{t h}^{*}, \mathrm{RCI}_{t h}\right)$ could equal the product of their marginal probabilities $\operatorname{POP}_{\tau}^{*}\left(\Delta k_{t h}^{*}\right) \times \operatorname{PRC}_{\tau}\left(\mathrm{RCI}_{t h}\right)$.

\section{Methodology \\ 3.1 Experimental}

The irradiance information used in this investigation was obtained from ground irradiance results measured at eight stations with differing latitudes, altitudes and microclimates. The specific locations of the stations are shown on a map in Figure 1, which also shows the respective location altitudes in metres.



Figure 1: Map showing the locations and altitude of the radiometric stations that provided irradiance data, where RVD = Ritchersveld , VAN = Vanrhynsdorp, GRT = Graaff-Reinet, NMU = Nelson Mandela Metropolitan University, UFS = University of Free State, UPR = University of Pretoria, VRY = Vryheid, and KZH = University of KwaZulu-Natal Howard College.

The station names that correspond to the acronyms displayed on the map are: Ritchersveld (RVD), Vanrhynsdorp (VAN), Graaff-Reinet (GRT), Nelson Mandela Metropolitan University (NMU), University of Free State (UFS), University of KwaZulu-Natal Howard College, (KZH), Vryheid (VRY) and University of Pretoria (UPR). They form part of Southern African Universities Radiometric Network (SAURAN), an initiative setup to provide high-resolution, ground-based radiometric data for Southern Africa $[13,14]$. The solar radiation components, global 
horizontal irradiance GHI, beam normal irradiance $\mathrm{BNI}$, and diffuse horizontal irradiance $\mathrm{DHI}$, are measured using state-of-the-art Kipp and Zonen [15] radiometers and are archived as one-minute-, hourly- and daily-averaged data. These data can be publicly accessed through a website interface [14]. A sample of one-minute-averaged solar irradiance data $(\Delta t=60 \mathrm{~s})$ measured over the year 01 July 2014-30 June 2015 was used. The BNI was converted to its horizontal surface component beam horizontal irradiance $\mathrm{BHI}$ through multiplication by the cosine of the solar zenith angle, i.e., $\mathrm{BHI}=\mathrm{BNI} \cos \theta_{\mathrm{z}}$. Daily time series of the solar resource features (Equations 2 and 3 ) were generated from this data sample using MATLAB Release 2011a. The solar utility indexes and the other parameters were computed for daily time intervals $\tau$ during 7:00 to 17:00 apparent solar time, with the fluctuation $\Delta k_{t h}^{*}$ and relative composition $\mathrm{RCI}_{\text {th }}$ thresholds set respectively at 0.01 and 0 . These daily probabilities are denoted by replacing the general time interval subscript $\tau$ by $\mathrm{D}$, i.e., $\mathrm{SUI}_{\mathrm{D}}, \mathrm{POP}_{\mathrm{D}}^{*}$ and $\mathrm{PRC}_{\mathrm{D}}$.

\subsection{SUI solar resource application to classification of solar resource}

The classification properties of the SUI are determined from a solar resource classifier built from the computed daily values of $\mathrm{SUI}_{\mathrm{D}}$ using $K$-means method to identify homogenous solar resource clusters. For a collection of $m, \mathrm{SUI}_{\mathrm{D}}^{n}$ data points where $n=1,2, \ldots m$, the $K$-means clustering algorithm iteratively groups the data points into $k$ disjoint clusters $C_{j}(j=1,2, \ldots k)$, each containing $m_{j}$ data points subject to minimisation of the within-cluster-sum-ofsquares error function [16]. The sum-of-square error is given by Equation 6 .

$$
\mathrm{E}=\sum_{\mathrm{j}=1}^{\mathrm{k}} \sum_{\mathrm{n} \in \mathrm{C}_{\mathrm{j}}}\left\|S U I_{\mathrm{D}}^{\mathrm{n}}-\mathrm{M}_{\mathrm{j}}\right\|^{2}
$$

where $\mathrm{M}_{\mathrm{j}}$ is the centre of the jth cluster, given by the mean of the data points belonging to the cluster. $\mathrm{A}$ collection of solar resource classification features consisting of a total $8 \times 365$ sample of daily solar utility indexes was used. To determine the number of clusters $k$ a distribution histogram of the SUID data visually identified the likely partitions of the data points. The $K$-means algorithm was applied to the data to create the clusters using a built-in function in the statistical toolbox of MATLAB software with the minimisation of the squared Euclidean distance as the clustering score. Measures known as silhouette values, $s\left(C_{j}, i\right)$, were calculated for each datum $i$ in each cluster $C_{j}$ using a built-in MATLAB function also named silhouette to determine the quality of the clusters. The silhouette values range from +1 indicating well-separated datum, through 0 for datum on the border of two clusters, to -1 for misclassified or outlier datum [17]. The cluster-specific averages $\bar{s}\left(C_{j}\right)$ measure how tightly grouped are all the data in the respective clusters. Values where $\bar{s}\left(C_{j}\right)>0.5$ were accepted to be representative of reasonably clustered data points.

\section{Results and discussion Solar utility index as a function of $P O P_{D}^{*}$ and $P R C_{D}$}

A somewhat moderate dependence exists between the marginal probabilities $P O P_{D}^{*}(0.01)$ and $\mathrm{PRC}_{\mathrm{D}}(0)$ as shown in Figure 2(a) and is characterised by a correlation coefficient of 0.65 . This probabilistic dependence is substantiated by Figure 2(b), which reveals a non-linear relation between joint probability $\operatorname{SUI}_{D}(0.01,0)$ and the product of the marginal probabilities $\operatorname{POP}_{D}^{*}(0.01) \times \operatorname{PRC}_{D}(0)$. The solid line in Figure 2(b), assumes independence of the marginal probabilities i.e. $\operatorname{SUI}_{\mathrm{D}}(0.01,0)=\operatorname{POP}_{\mathrm{D}}^{*}(0.01) \times$ $\mathrm{PRC}_{\mathrm{D}}(0)$; and shows that this assumption generally underestimates the SUI . The mean bias error and root mean square error relative to the sample mean $\mathrm{SUI}_{D}$ associated with this assumption for this sample of data are $-5.1 \%$ and $9.2 \%$, respectively. A quadratic fit as shown by the broken line on the same graph gives a better fit with coefficient of determination value $R^{2}=0.99$ and a root mean square error relative to mean $\mathrm{SUI}_{\mathrm{D}}$ of $5.8 \%$.

\subsection{Variation with $\Delta k_{t h}^{*}$, and $R C I_{\text {th }}$}

The variation of SUI with $\Delta k_{t h}^{*}$, and $\mathrm{RCI}_{t h}$ can be indirectly inferred from the daily Cumulative Distribution Functions, $\mathrm{F}_{\left|\Delta k_{T}^{*}\right|}\left(\Delta k_{t h}^{*}\right)$ and $\mathrm{F}_{\mathrm{RCI}}\left(\mathrm{RCI}_{t h}\right)$. Figure 3 illustrates these functions for three solar resource profiles that exhibit different characteristics. It was found that, for $\mathrm{PRC}_{D}>0$, $\mathrm{SUI}_{D}$ increases with the fluctuation threshold $\Delta k_{\text {th }}^{*}$ since $\operatorname{POP}_{\mathrm{D}}^{*}\left(\Delta k_{t h}^{*}\right)=\mathrm{F}_{\left|\Delta k_{T}^{*}\right|}\left(\Delta k_{t h}^{*}\right)$ is a monotonically increasing function of $\Delta k_{t h}^{*}$ despite the error associated with estimating the $\mathrm{SUI}_{D}$ via $\mathrm{POP}_{\mathrm{D}}^{*} \times \mathrm{PRC}_{D}$. By examining the $\mathrm{F}_{\mathrm{RCI}}\left(\mathrm{RCI}_{\text {th }}\right)$ curve we also similarly observe that for $\mathrm{POP}_{\mathrm{D}}^{*}>0$, increasing $\mathrm{RCI}_{t h}$ results in higher values of $\mathrm{PRC}_{D}$ hence higher SUID. The solar utility indexes can be computed for shorter time intervals $\tau$, such as hourly intervals, or longer time intervals $\tau$, such as months, as long as the irradiance data sampling time interval $\Delta t$, allows for large enough sample sizes, $N=\tau / \Delta t$. Longer sampling time intervals may, however, mask the effect of the short-term solar resource variability. 
(a)



(b)



Figure 2: (a) Relationship between the marginal probabilities $\operatorname{POP}_{D}^{*}(0.01)$ and $\mathrm{PRC}_{\mathrm{D}}(0)$. (b) Relationship between SUlD $(0.01,0)$ and the product of marginal probabilities $\operatorname{POP}_{D}^{*}(0.01) \cdot \operatorname{PRC}_{D}(0)$.

(a)

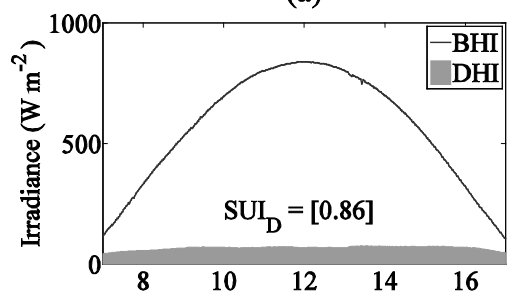

(b)

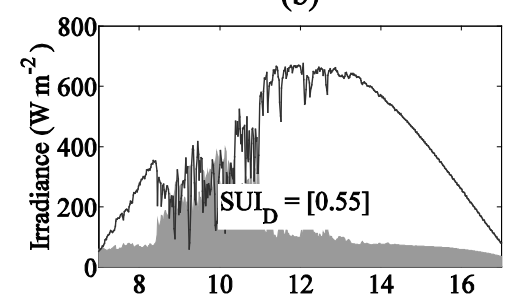

(c)

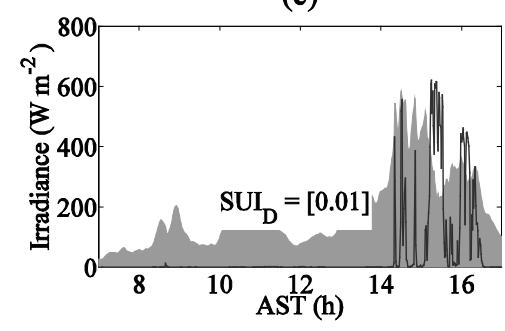

(d)

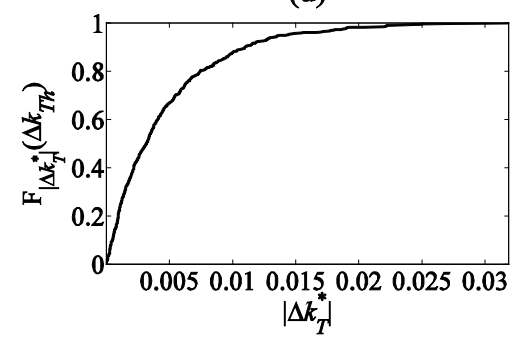

(e)



(f)

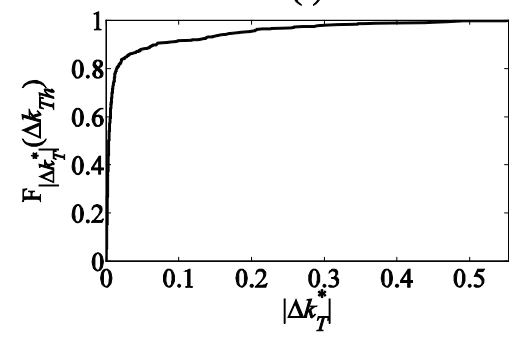

(g)

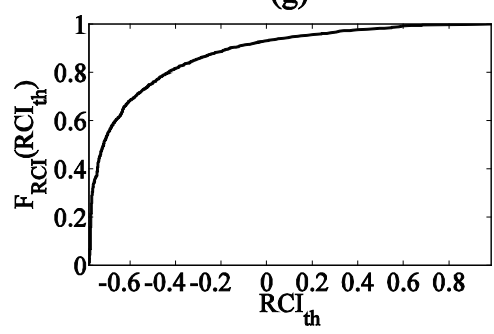

(h)

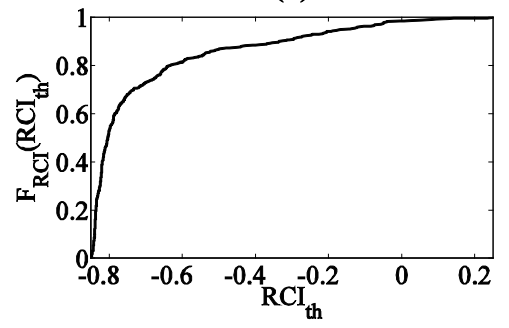

(i)



Figure 3: (a)-(c) Typical daily solar resource component (BHI and DHI) time series for different days showing different solar resource variability as a function of apparent solar time. Solar utility index associated with each solar resource profile is displayed on each corresponding figure. The cumulative distribution curves matching the solar profiles are shown in subplots (d)-(f) for the solar resource fluctuation magnitude and in subplots (g)-(i) for the relative composition index. 


\subsection{The SUI $I_{D}$ solar resource classification qualities}

This section demonstrates the solar resource classification qualities of the SUI . A sample of $365 \times 8$ values of $\mathrm{SUI}_{\mathrm{D}}$, generated using the following parameters: $\Delta k_{t h}=0.01, \mathrm{RCI}_{t h}=0, \Delta t=1 \mathrm{~min}$ and $\tau=$ 7:00-17:00 apparent solar time, was considered.

\section{Clustering results}

Figure 4(a) shows a histogram of the distribution of the sample $\mathrm{SUI}_{\mathrm{D}}$ values for all stations, from which one can identify the following five cluster-definingboundaries: $\mathrm{SUI}_{\mathrm{D}} \geq 0.8,0.6 \leq \mathrm{SUI}_{\mathrm{D}}<0.8,0.4 \leq$ $\mathrm{SUI}_{\mathrm{D}}<0.6,0.2 \leq \mathrm{SUI}_{\mathrm{D}}<0.4$, and $\mathrm{SUI}_{\mathrm{D}}<0.2$; labelled cluster 1 to 5 respectively. The quality of each of these clusters is shown by the silhouette plot in Figure 4(b). A small percentage of its population of about $4 \%$ is misclassified as indicated by the negative silhouette values, despite the largest cluster-averaged silhouette values recorded by cluster 5 pointing to a good clustering. Some misclassified data representing $1.3 \%, 2.4 \%$ and $0.25 \%$ of the respective cluster populations was also shown in clusters 1 , 2 and 3 . Applying the $K$-means, clustering method shows an improvement in the data clustering as shown in the silhouette plot of Figure 4(c). Cluster 5 again appeared to be the best clustered. Cluster 3 and 4 show some data points that have negative silhouette values, but constitute only $1.8 \%$ and $1.1 \%$ of the respective cluster populations.

\section{Interpreting the clusters}

Figure $5(\mathrm{a})$ shows the clustered $\mathrm{SUI}_{\mathrm{D}}$ as a function of $\mathrm{CSI}_{D}$, the daily-averaged value instantaneous CSI. The results show a positive correlation between the $\mathrm{SUI}_{\mathrm{D}}$ and $\mathrm{CSI}_{\mathrm{D}}$ following an exponential relationship as shown by the solid line that traces the trend

(a)



of cluster centroid on the graph. The results also show a spread of data points around these cluster centroids and the extent of these dispersions vary as shown in Figure 5(b) by the sample standard deviations of the cluster $\mathrm{CSI}_{D}$ and $\mathrm{SUI}_{\mathrm{D}}$. Cluster 5 is the least compact with largest spread of $\mathrm{CSI}_{D}$. It is conceivable that 2 smaller and more compact clusters can be obtained by splitting cluster 5 along the line $\mathrm{CSI}_{\mathrm{D}}=0.6$, illustrated by the thick broken vertical line in Figure 5(a). Clusters 1 and 2 appear to be the most compact clusters judging from the spread of their $\mathrm{CSI}_{\mathrm{D}}$ and $\mathrm{SUI}_{\mathrm{D}}$ values.

Further characteristics of the five clusters are demonstrated in in Figures 6(a) and (b), which show the $\mathrm{SUI}_{\mathrm{D}}$ as a function of daily-averaged fluctuation magnitude $\left\langle\left|\Delta k_{T}\right|\right\rangle_{\mathrm{D}}$ and daily-averaged relative composition index $\mathrm{RCI}_{\mathrm{D}}$ respectively. There is a general increase in solar resource $\left\langle\left|\Delta k_{T}\right|\right\rangle_{\mathrm{D}}$ from cluster 1 to cluster 4 . Cluster 5 shows a slight deviation from this trend, an indication of significant population of low fluctuation cloudy-sky solar resource within this cluster. The dispersion of the fluctuation magnitudes within each cluster also tended to increase with the cluster number as shown by the cluster-specific standard deviations in Figure 6(c). Figure 6 (b), while recalling that the $\mathrm{RCI}_{\mathrm{D}}$ is an indication of the balance between the $\mathrm{DHI}$ and $\mathrm{BHI}$, reveals that $\mathrm{BHI}$ dominated the solar resource belonging to clusters 1 to $3\left(\mathrm{RCI}_{\mathrm{D}}<0\right)$. Cluster 4 , having and average $\mathrm{RCI}_{\mathrm{D}}$ close to 0 , appears to be evenly populated by both BHI dominated, and DHI dominated, solar resource. An additional cluster is conceivable from splitting cluster 4 along the $\mathrm{RCI}_{\mathrm{D}}=0$. The withincluster standard deviations of $\mathrm{RCI}_{D}$ are shown in Figure 6(c) and show a general increase with cluster number.

(b)

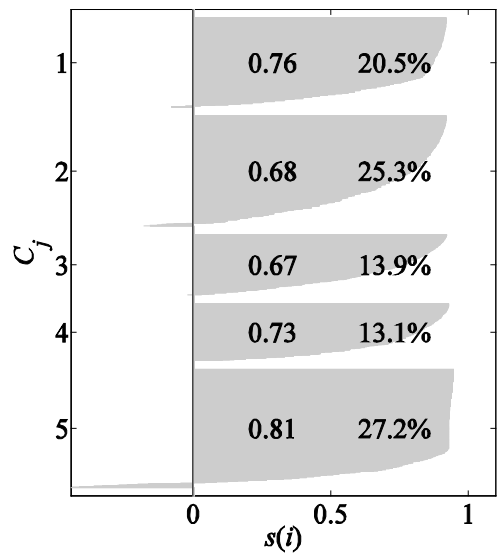

(c)



Figure 4: (a) Histogram showing the distribution of the SUlD values and; Figures 4(b) and (c) are Silhouette plots of the classifications obtained using; boundary SUlD ranges identified from the Figure 4(a) and, $K$-means clustering algorithm respectively. The numbers displayed to two decimal places and as percentages on each cluster plot show cluster-specific, average silhouette value $\bar{s}\left(C_{j}\right)$, and percentage population, respectively. 
(a)

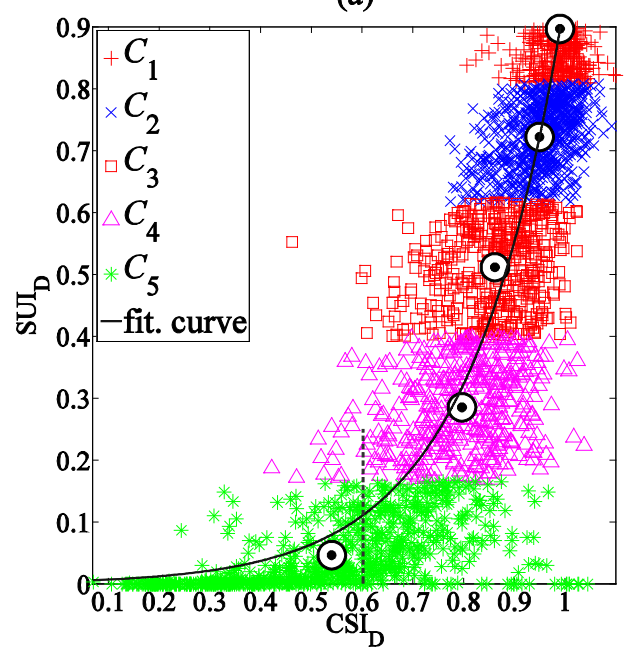

(b)

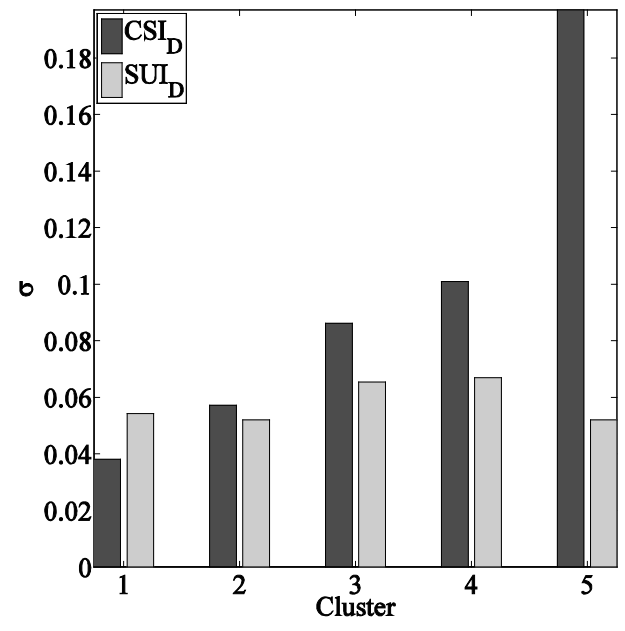

Figure 5: (a) Clustered SUID as a function of clear-sky index CSID and (b) cluster-specific relative standard deviation of CSID and SUID variation among clusters. $C 1$ to $C 5$ are the clusters identified by $K$ means clustering of the SUlD. The coordinates of the cluster centroids, marked by $\odot$, are defined by the average of the respective cluster SUID and CSID.

(a)

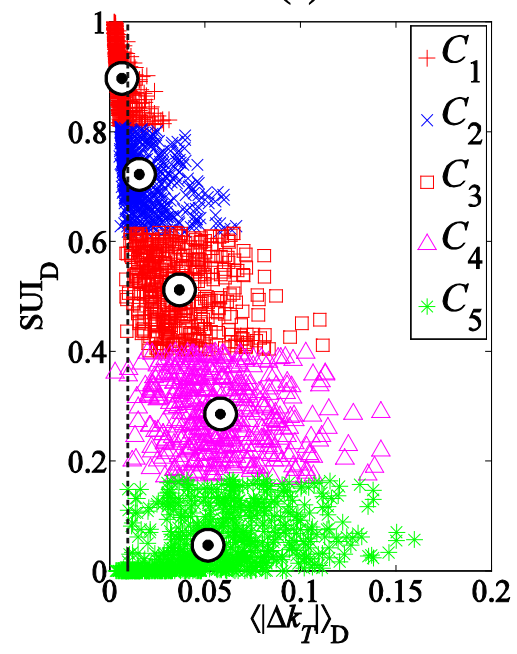

(b)

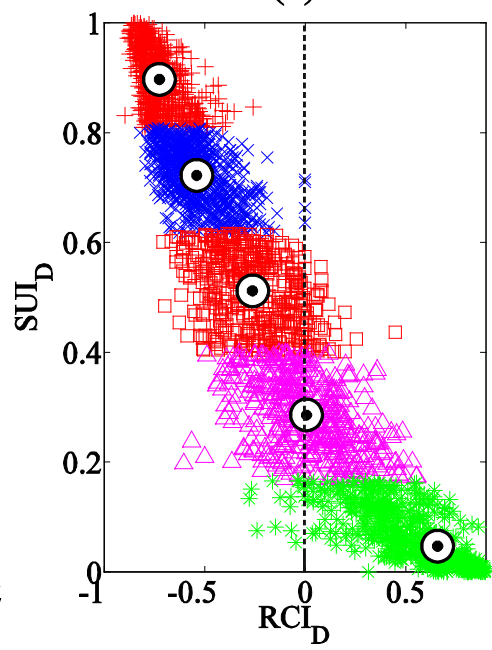

(c)

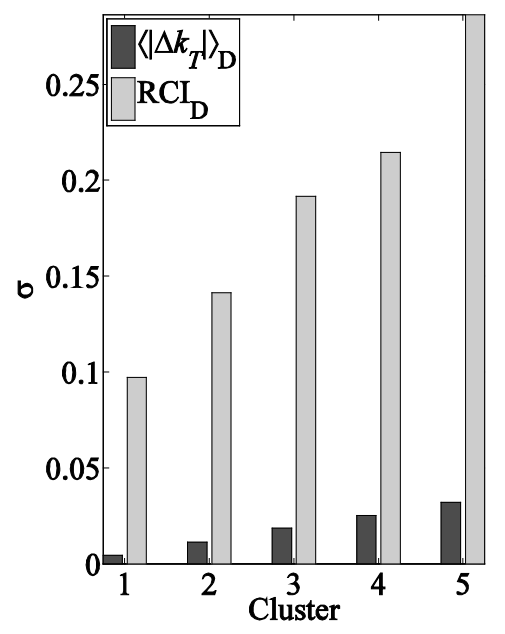

Figure 6: Clustered SUlD as a function of (a) daily averaged absolute solar resource fluctuation magnitude $\left\langle\left|\Delta k_{T}\right|\right\rangle_{D}$ and (b) Daily Relative Composition Index RClD; (c) cluster specific standard deviations of the fluctuation magnitude and the relative composition index. The vertical broken lines in (a) and (b) represent the threshold values used to define the SUID, $\Delta k_{t h}^{*}=0.01$, and $\mathrm{RCl}_{t h}=0$ respectively. The coordinates of the cluster centroids, marked by $\odot$, are defined by the average of the respective cluster variables.

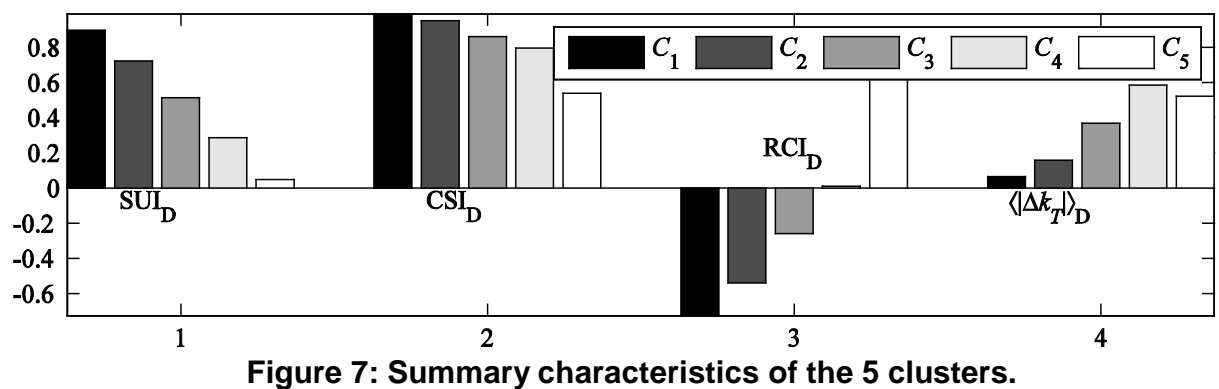



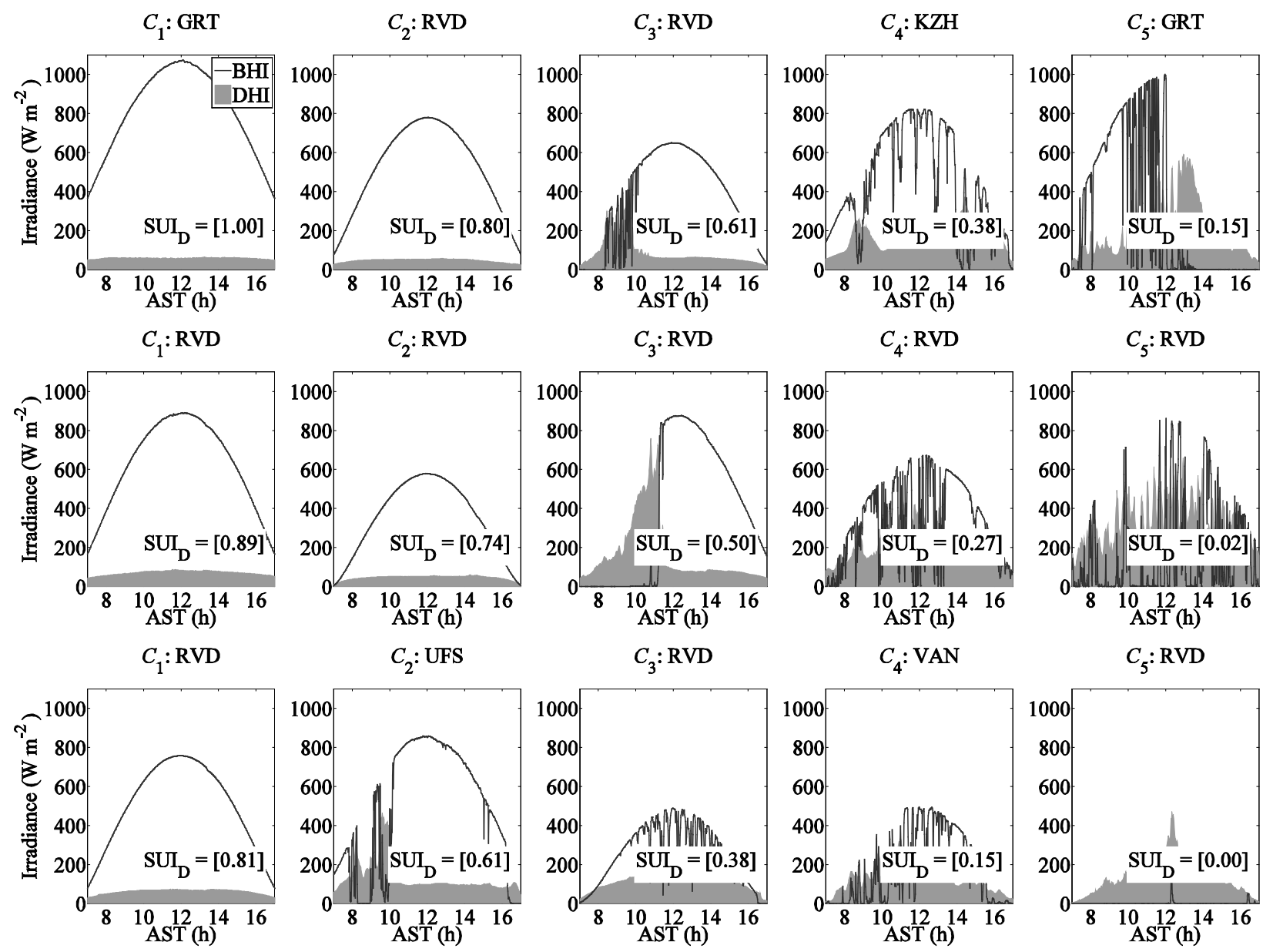

Figure 8: Samples of diurnal profiles of horizontal; beam, and diffuse irradiance belonging to, from left to right, cluster 1 through 5 and sampled, top to bottom, at the within-cluster maximum, median and minimum values of the respective cluster SUlD. The graphs also show the SUlD that is characteristic of each of the selected day's solar resource.

Figure 7 gives a summary of the characteristic statistics of the clusters in terms of the mean values of $\mathrm{SUI}_{\mathrm{D}}, \mathrm{CSI}_{\mathrm{D}}, \mathrm{RCI}_{\mathrm{D}}$ and $\left\langle\mid \Delta k_{T}\right\rangle_{\mathrm{D}}$. Figure 8 shows typical solar resource diurnal profiles sampled from each cluster at: maximum, median, and minimum $\mathrm{SUI}_{\mathrm{D}}$ values. The profiles vary across the clusters in amplitude as well as the frequency and duration of cloud induced discontinuities. The trends of these variations correspond to the trends of the summary statistics depicted in Figure 7. For example, cluster 4 profiles shown in Figure 8 appear to have the highest frequency of discontinuities in agreement with mean value of $\left\langle\left|\Delta k_{T}\right|\right\rangle_{D}$, which is also largest for cluster 4 as shown in Figure 7. It is also noted that the solar resource profiles at the shared boundaries of the clusters show similar properties.

\section{Cluster variation amongst stations}

The observed varying of the five clusters was also investigated across the eight stations. Figure 9 shows silhouette plots of the five clusters for each station.
The results reveal a distribution of cluster populations that varied across the stations and appeared to be a function of site specific climatic conditions. For example, the solar resources at NMU and $\mathrm{KZH}$ were dominated by cluster 5-type solar resource, which represents the lowest values of the SUI, hence an indication of high prevalence of cloudy-sky conditions. The NMU and $\mathrm{KZH}$ are located in coastal cities of Port Elizabeth and Durban, respectively, and were characterised by sky conditions that are cloudy, or with shade, haze or low sun intensity for $37.5 \%$ and $46.5 \%$ of the possible sunshine hours, respectively [18]. The RVD, VAN, UFS and UPR, on the other hand, appeared to have a higher prevalence of clear-sky periods as shown by the larger populations of data points in clusters 1 and 2 .

\section{Conclusions}

This paper proposes and presents a new solar resource metric named solar utility index that measures the fractional time when a solar resource 

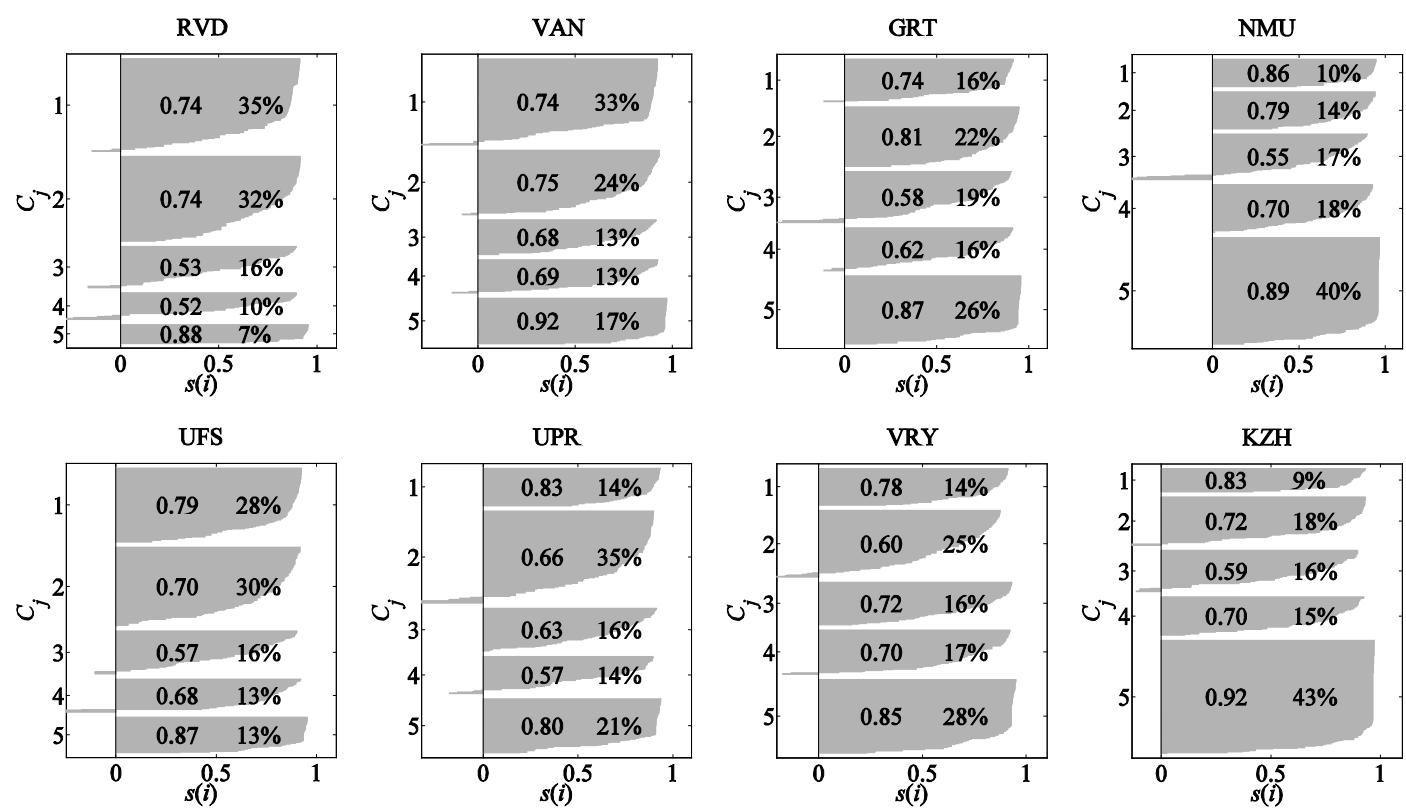

Figure 9: The clusters identified by the $K$-means algorithm for each of the eight stations.

has short-term fluctuation magnitudes and energy quality that satisfy given or set conditions during a time period $\tau$ within a solar resource time span from sunrise to sunset. Five clusters were identified and were found to have reasonably homogeneous intracluster properties, in terms of energy content $\mathrm{CSI}_{D}$, short-term variability $\left\langle\left|\Delta k_{T}\right|\right\rangle_{\mathrm{D}}$, and relative $\mathrm{DHI}$ and $\mathrm{BHI}$ composition $\mathrm{RCI}_{\mathrm{D}}$. A closer look at the cluster properties, however, revealed that clusters 5 and 4 can be split into smaller clusters by taking into account the distribution of their $\mathrm{CSI}_{\mathrm{D}}$ and $\mathrm{RCI}_{\mathrm{D}}$, respectively. The theoretical basis of the SUI suggests that it can be computed for periods longer or shorter than the 10 hour daily period considered. It is therefore important to investigate its solar resource classifying properties for periods shorter or longer than the daily period. It is also interesting to investigate how the SUI performs as a solar resource forecasting metric.

\section{Acknowledgements}

The author wishes to acknowledge the Southern African Universities Radiometric Network as the source of solar radiation data used in this work.

\section{References}

1. Twidell, J. and Weir, T. 2006. Renewable energy resources, 2nd edition. Taylor and Francis.

2. Duffie, J. and Beckman, W. 2013. Solar engineering of thermal processes. 4th edition. John Wiley and Sons.

3. Peled, A. and Appelbaum, J. 2013. Evaluation of solar radiation properties by statistical tools and wavelet analysis. Renewable Energy 59: 30-38. http://dx.doi.org/10.1016/j.renene.2013.03.019.
4. Maafi, A. and Harrouni, S. 2003. Preliminary results of the fractal classification of daily solar irradiances. Solar Energy 75: 53-61. http://dx.doi.org/ 10.1016/S0038-092X(03)00192-0.

5. Soubdhan, T., Emilion, R. and Rudy, C. 2009. Classification of daily solar radiation distributions using a mixture of dirichlet distributions. Solar Energy 83: 1056-1063.

http://dx.doi.org/10.1016/j.solener.2009.01.010.

6. Gastón-Romeo, M., Leon, T., Mallor, F. and Ramírez-Santigosa, L. 2011. A morphological clustering method for daily solar radiation curves. Solar Energy 85: 1824-1836. http://dx.doi.org/10.1016/ j.solener.2011.04.023.

7. Stein, J. S., Hansen, C. W. and Reno, M. J. The variability index: A new and novel metric for quantifying irradiance and PV output variability. World Renewable Energy Forum, Denver, CO, 2012.

8. Kang, B. O. and Tam, K. 2013. A new characterization and classification method for daily sky conditions based on ground-based solar irradiance measurement data. Solar Energy 94 :102-118. http://dx.doi.org/10.1016/j.solener.2013.04.007.

9. Perez, R., Kivalov, S., Schlemmer, J., Hemker Jr, K. and Hoff, T. 2011. Parameterization of site-specific short-term irradiance variability. Solar Energy, 85 : 1343-1353. http://dx.doi.org/10.1016/j.solener. 2011.03.016.

10. Ineichen, P. and Perez, R. 2002. A new airmass independent formulation for the linke turbidity coefficient. Solar Energy, 73: 151-157. http://dx.doi.org/10.1016/S0038-092X(02)00045-2.

11. SNL. 2012. Pv_lib toolbox for matlab. Sandia National Laboratories. Available from: http://pvpmc.org/ pv-lib/ [Accessed: 02 January 2015].

12. Kalogirou, S. A. 2014. Chapter 11 - designing and modeling solar energy systems. In: Kalogirou, S. A. (ed.) Solar energy engineering, second edition. Boston: Academic Press, 583-699. http://dx.doi.org/ 10.1016/B978-0-12-397270-5.00011-X. 
13. SAURAN. 2015. Southern African Universities Radiometric Network. Available from: http://www.sauran.net/ [Accessed: 10 October 2015].

14. Brooks, M. J., du Clou, S., van Niekerk, W. L., Gauché, P., Leonard, C., Mouzouris, M. J., Meyer, R., van der Westhuizen, N., van Dyk, E. E. and Vorster, F. J. 2015. Sauran: A new resource for solar radiometric data in Southern Africa. Journal of Energy in Southern Africa 26: 2-10.

15. Kipp and Zonen. 2017. Kipp \& zonen pyranometers. Available from: http:/www.kippzonen.com/ProductGroup/3/Pyranometers [Accessed: 17 May 2017].

16. Nabney, I. T. 2002. Netlab: Algorithms for pattern recognition. In: Singh, S. (ed.) Advances in pattern recognition. Great Britain: Springer.

17. Rousseeuw, P. J. 1987. Silhouettes: A graphical aid to the interpretation and validation of cluster analysis. Journal of Computational and Applied Mathematics, 20: 53-65. http://dx.doi.org/10.1016/03770427(87)90125-7.

18. Climatemps. 2014. Sunshine \& daylight hours in Durban, South Africa. Climatemps. Available from: http://www.durban.climatemps.com/sunlight.php [Accessed: 27 January 2016]. 